\title{
Usages Sociaux Des Rameaux Et Exploitation Durable De Raphia Sudanica Dans Le Sud-Est Du Benin
}

\author{
Dr Ludovic K. N'TCHA ${ }^{1 *}$, Dr Monique OUASSA KOUARO ${ }^{2}$ \\ ${ }^{I}$ Université d'Abomey-Calavi \\ ${ }^{2}$ Associate Professor CAMES Université d'Abomey-Calavi
}

*Corresponding Author: Dr Ludovic K. N'TCHA, Université d'Abomey-Calavi

\begin{abstract}
The communities of southeastern Benin use the different organs of Raphiasudanica for social, medicinal, ritual, cultural, religious, etc. purposes. As a result, this species is subject to anthropic pressure and is at risk of extinction in this environment. The objective of this research is to describe the social uses of the branches of Raphiasudanica which influence its sustainable exploitation in the South-East of Benin. It adopted a qualitative approach and relied on in-depth interviews, group discussions, direct observations and documentary analysis. The symbolic interactionism of $H$. Blumer revisited by $L$. Lacaze is the theoretical model used for the analysis of the results. The results reveal that the branches of Raphiasudanica are used by local social groups for economic, religious, medicinal and security purposes. The users of these organs are not concerned about the way in which the branches are used in the environment. The degree of destruction of a tree species is a function of its value to local social groups in the vicinity or the multiplicity of its use by them. In Western Cameroon, raffia palm groves are the raw material of craftsmen and a whole civilization, which feeds on raffia wine and articles made from raffia palm groves, is gradually disappearing. The social uses of the twigs from this plant thus contribute to its progressive destruction because its exploitation is not such as to ensure its sustainability.
\end{abstract}

Keywords: Raphiasudanica, social uses, twigs, sustainable exploitation, Benin.

\section{INTRODUCTION}

La dégradation ou du moins la disparition d'une espèce environnementale n'est pas toujours due àl'effet de fin de cycle de sa vie. Plusieurs espèces vivantes de l'ordre animal ou végétal ont disparu ou sont en voie de disparition en raison des effets anthropiques. Les pollutions environnementales de tout genre, les feux de végétations, la déforestation et plus encore les exploitations incontrôlées de ces espèces sont entre autres les causes majeures d'extinction des espèces animales et végétales.

En ce qui concerne les forêts, une revue générale de la littérature, une synthèse biographique spécifique et les avis d'experts ont permis d'identifier d'un point de vue théorique, les causes de la déforestation et la dégradation des forêts. Ces variables potentielles ont été regroupées en 9 sousensembles : l'agriculture, les infrastructures, l'exploitation forestière, les facteurs économiques, les axes de transport, les facteurs démographiques, les facteurs socioculturels, les facteurs institutionnels et les facteurs biophysiques (C. Ernst et al., 2012). Ces constats mettent en exergue plusieurs raisons explicatives de la dégradation du couvert végétal etdont les sources sont directement associées aux activités humaines. Plusieurs institutions militant en faveur de l'environnement ont mis l'accent à mainte reprise sur l'impact négatif de la déforestation sur les ressources naturelles en général et sur le couvert végétal en particulier. Il n'est donc d'aucun doute que «la déforestation représente une sérieuse menace à la durabilité environnementale et met en péril les progrès accomplis vers l'éradication de la pauvreté et de la faim » (PNUD, 2013, p. 42).

En effet, le rapport de l'Homme à la nature,qu'on peut situer à la naissance de l'humanité,n'a fait qu'évoluer dans le temps et dans l'espace. L'espèce humaine a toujours usé de son intelligence pour exploiter les ressources naturelles qui l'entourent et cette remarque fonde l'interaction entre «nature et culture » qu'évoque P. Descola (2015). Les palmiers sauvages quoi que classés dans les produits forestiers non ligneux (PFNL), sont en proie au même titre que les autres espèces forestières. D'ailleurs, «les populations locales utilisent quotidiennement et en majorité des produits forestiers 
non ligneux (PFNL) en vue de satisfaire leurs besoins »(Dongmo, n.d., p. 9). Les raphiales constituent la matière première des artisans ; on y extrait du vin de raphia, et des articles sont fabriqués à partir des organes tirés de ces raphiales, qui, il faut le souligné, disparaitt progressivement (L. Meutchieye, 2012).On comprend donc que «les PFNL sont généralement utilisés pour compléter les régimes alimentaires et augmenter le revenu des ménages, notamment pendant des saisons particulières de l'année telles que les saisons sèches, et pour aider à répondre aux besoins médicinaux » (J. E. Michel Arnold et M. Ruiz Perez, 2001, p. 440).

Ces divers usages sociaux du Raphia sudanica ont un impact sur son exploitation durable. En effet, dans le sud-est du Bénin, les groupes sociaux locaux ont recours aux organes de cette espèce dans leurs pratiques quotidiennes. C'est le cas des usages sociaux des rameaux de cette espèce, qui sont utilisés à plusieurs fins et méritent que l'on s'y intéresse pour une appréhension de leur impact sur la conservation de l'espèce dans le Sud-Est du Bénin. Parce que, les usages sociaux prouvent parfois l'attachement des groupes sociaux à une ressource particulière et relève au passage les rapports sociaux qu'ils entretiennent avec la nature. Il y a donc lieu de se poser la question de savoir : quels sont les défis liés à l'exploitation durable des rameaux de Raphia sudanica? Cette préoccupation a donné une opportunité d'apporter des réponses scientifiques à travers la description des usages sociaux des rameaux de Raphia sudanica qui impactent son exploitation durable dans le Sud-Est du Bénin. Certes, les rameaux de cette espèce ne sont pas les seuls auxquels ont recours les groupes sociaux locaux, mais il convient de signaler que l'accent est particulièrement mis sur l'usage des rameaux de cette plante dans la présente recherche.

\subsection{Matériel Et Méthodes}

Le cadre de cette recherche couvre la partie méridionale du Bénin, précisément dans le Sud-Est qui couvre principalement deux départements, l'Ouémé et le Plateau (Figure 1). Ces départements comptent respectivement 05 communes (Kétou, Pobè, Adja-Ouèrè, Sakété et Ifangni) pour le Plateau, et 09 communes (Adjara, Akpro-Missérété, Avrankou,Adjohoun, Bonou, Dangbo, Sèmè-Kpodji, Aguégué et Porto-novo) pour l'Ouémé (INSAE, 2013).

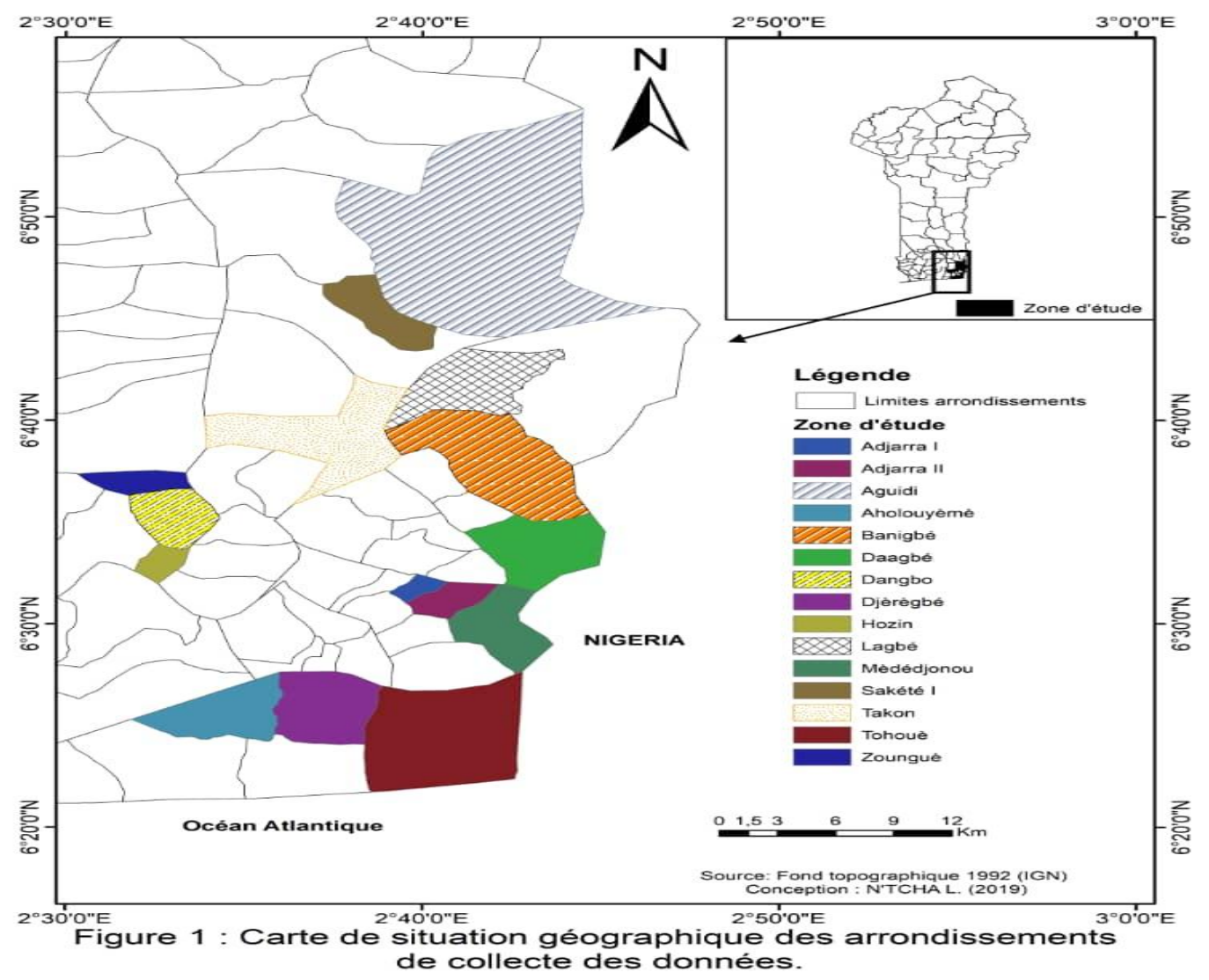

Source: Tabé et N'Tcha, 2020

Les 14 communes de ces départements n'ont pas été toutes enquêtées lors de la collecte des données de terrain. Les départements de l'Ouémé et du Plateau comptent respectivement neuf (09) et cinq (05) 
communes ; ce qui fait, au total, quatorze (14) communes. La technique de choix raisonné a permis de retenir les localités dans lesquelles les données ont été collectées. Cinqcommunes, à raison de trois (03) dans le département de l'Ouémé et deux (02) dans le département du Plateau ont été retenues. C'est donc le tiers (1/3) représentatif des communes de chacun de ces départements par excès, qui a servi de zone de collecte des données des informations relatives au Raphia sudanica. Les Communes de Sèmè-Podji, d'Adjarra et de Dangbo ont été retenues dans le département de l'Ouémé et celles d'Ifangniet de Sakété dans le Plateau. Parce que, dans ces communes, on retrouve plus les exploitants de Raphia sudanica. Les critères qui ont orienté le choix des communes, des arrondissements, les quartiers de villes ou villages sont : la présence des bas-fonds qui regorgent de raphiales, des artisans qui travaillent les organes de l'espèce, des exploitants du vin de raphia et l'observation de toute autre forme d'usage des organes de Raphia sudanica. A cela il faut ajouter que les principaux groupes sociolinguistiques autochtones que sont: les Gun, les Tori, les Xwla et les Nago-Yoruba, vivent majoritairement dans les localités retenues.

En ce qui concerne le nombre d'acteurs interrogés par groupe cible, il a été déterminé à partir d'un échantillonnage basé sur la technique de choix raisonné. Le choix des groupes cibles approchés s'est opéré de façon raisonnée sur la base des informations recherchées. Par conséquent, à partir de la technique de choix raisonné, neuf (9) groupes cibles composés de : dignitaires religieux, sages et notables, tradi-thérapeutes, artisans, les femmes, pêcheurs, conservateurs de musée, exploitant de vin de Raphia sudanicaet représentants des institutions gouvernementales ou non gouvernementales.

Les dignitaires religieux ont fourni les informations sur l'exploitation et les usages sociaux des rameaux lors de certainsrituelsreligieux. Les sages et notables ont révélé l'utilité des rameaux de l'espèce, l'évolution de leurs usages à travers le tempsetles valeurs et normes que représentent les rameaux de Raphia sudanica pour les groupes sociaux locaux. Les tradi-thérapeutes ont ressorti les vertus thérapeutiques des rameaux de cette plante et les circonstances dans lesquelles ils recourent à eux. Quant aux artisans, ils ont expliqué pourquoi les rameaux sont de plus en plus sollicités dans le milieu artisanal et les enjeux de la gestion durable de cette espèce dans leur milieu. Les pêcheurs ont évoqué l'intérêt de l'usage des rameaux de cette plante pour la pêche. Enfin, les représentants des institutions gouvernementales et non gouvernementales ont donné des précisions quant aux actions menées dans le cadre de la préservation du couvert végétal dans ces départements (Ouémé et Plateau), les résultats de ces actions, ainsi que les difficultés auxquelles est confrontée l'application des textes relatifs à l'exploitation des ressources forestières.

Cette technique a été choisie en raison de la nature des données collectées, mais aussi du fait que seule une catégorie d'acteurs répondant aux critères prédéfinis a été interrogée. Les critères de choix des interlocuteurs sont: l'âge, le sexe, la durée de vie de l'individu dans le milieu,l'appartenance à un groupe sociolinguistique du milieu. Pour l'âge, trois catégories ont été définies suivant le principe utilisé par A. E. Assogbadjoet al. (2012) pour les études ethnobotaniques. Il s'agit des jeunes (âge < 30 ans), des adultes (âge compris entre 30 et 60 ans) et des vieux (âge > 60 ans). Ces différentes tranches d'âge couvrent toutes les catégories d'individus d'un groupe social (enfants, adultes et personnes du troisième âge), qui n'ont pas toujours les mêmes rapports avec le milieu naturel (A. E. Assogbadjoet al.,2012).

Au total, 27 interlocuteurs ont été approchés et cette taille de l'échantillon a été arrêtée suivant le principe d'atteinte du seuil de saturation des informations. Les techniques de collecte des données mobilisées sont l'analyse documentaire à partir d'une fiche de lecture, l'entretien individuel approfondi et de groupe opérationnalisés avec des guides d'entretien, l'observation directe au moyen d'une grille d'observation. L'analyse de contenu a permis de faire le tri thématique des données. Les résultats issus du traitement des données collectées ont été analysés suivant la théorie de l'interactionnisme symbolique de Blumerrevisité par L. Lacaze (2013).

\section{RESUltats}

Le traitement des données a révélé plusieurs usages sociaux des rameaux de Raphia sudanica. Ces différents usages ont été catégorisés suivant les fins auxquelles ils sont destinés. De ce fait, on observe les usages des rameaux à des fins économiques, thérapeutiques, magico-religieuses et sécuritaires.

\subsection{Usages Sociaux Des Rameaux a Des Fins Economiques}

Les rameaux de Raphia sudanica sont utilisés par les artisans pour la fabrication de plusieurs objets destinés à la commercialisation. Les artisans usent des rameaux de diverses manières pour la 
confection de ces objets. Par conséquent, les rameaux interviennent dans la fabrication des sacs, des nattes, des balayes, des cordes, des toits, des vêtements, des accoutrements de divinités, des objets décoratifs.

En dehors des accoutrements de divinités que l'on retrouve exclusivement dans les couvents, les autres objets fabriqués avec les rameaux ou extraits des rameaux de Raphia sudanicasont commercialisés à domicile, dans les marchés locaux et accessibles à tous les usagers. En effet, plusieurs types de nattes sont tressés avec les fibres issues des rameaux de cette plante.

Il faut préciser que les femmes s'intéressent particulièrement aux organes de Raphia sudanicaen général et à ses rameaux en particulier, pour entretenir leurs activités génératrices de revenus. En effet, environ 37,64\% des artisans approchés de ces localités sont des femmes vannières, tresseuses de cordes et de pailles, fabricantes du vin de raphia, connue sous le nom de «dehán $»^{1}$ (en langue locale Gun) pour ne citer que ces activités-là. Elles y sont très actives dans ces activités qui leur permettent de s'affirmer financièrement et même de faire des épargnes. Une artisane déclare que « les organes de raphia offrent aux femmes de notre localité, l'opportunité de se rendre autonome financièrement, de contribuer à la vie économique de son foyer et d'épargner pour d'autres besoins éventuels » (Propos de : AH, Adjarra, 08/06/2019). Ce verbatim montre l'impact des usages sociaux de cette plante sur la vie économique des groupes sociaux locaux, mais aussi l'opportunité d'autonomisation qu'elle offre aux femmes du Sud-Est du Bénin. La planche suivante présente les activités génératrices de revenus exercées par les femmes grâce aux organes de Raphia sudanica.

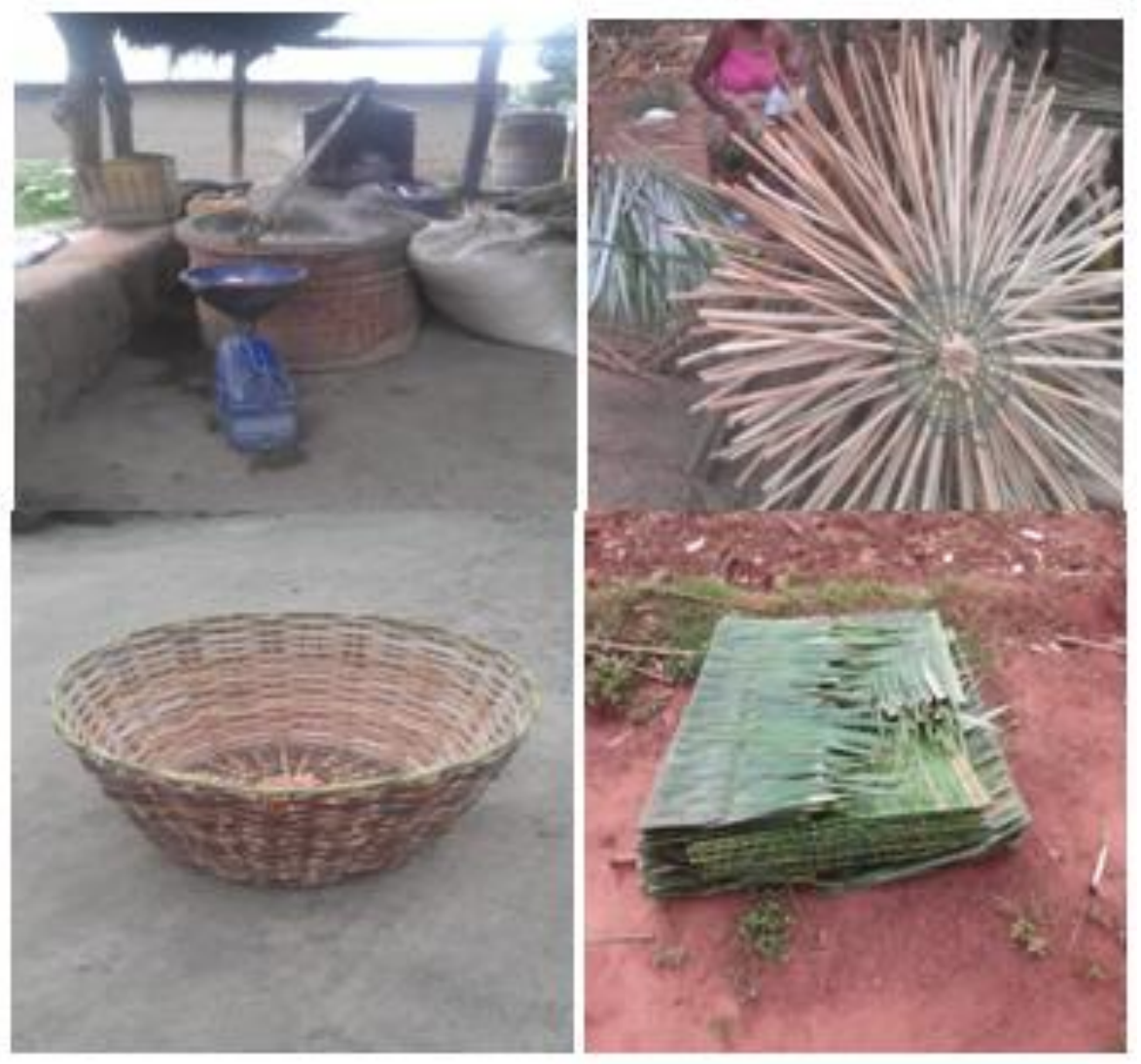

Planche1. Usages de Raphia sudanicadans les activités génératrices de revenus des femmes

Source. Cliché N'Tcha, 2019

Le verbatim suivant, tiré du discours d'un enquêté stipule que : « la natte « kpãgnã » sur laquelle les rois s'assaillent, est une natte tressée à partir des rameaux et de leurs fibres. Ce sont les rameaux de Raphia sudanica et leurs fibres qui permettent de la fabriquer» (Propos de: HL, Kétonou le 08/06/2019). Ce verbatim soutient l'usage des rameaux de cette espèce dans la fabrication des nattes qu'elles soient ordinaires ou sacrées. La photo ci-dessous présente les fibres issues des rameaux auxquelles ont recours les artisans au besoin pour compléter la fabrication des objets.

\footnotetext{
${ }^{1}$ Boisson connue sous le nom de "sodabi" mais fabriquée à partir du vin extrait de Raphia sudanicaà ne pas confondre avec le "sodabi" obtenue à partir du vin issu du palmier à huile, le « déhán ».
} 


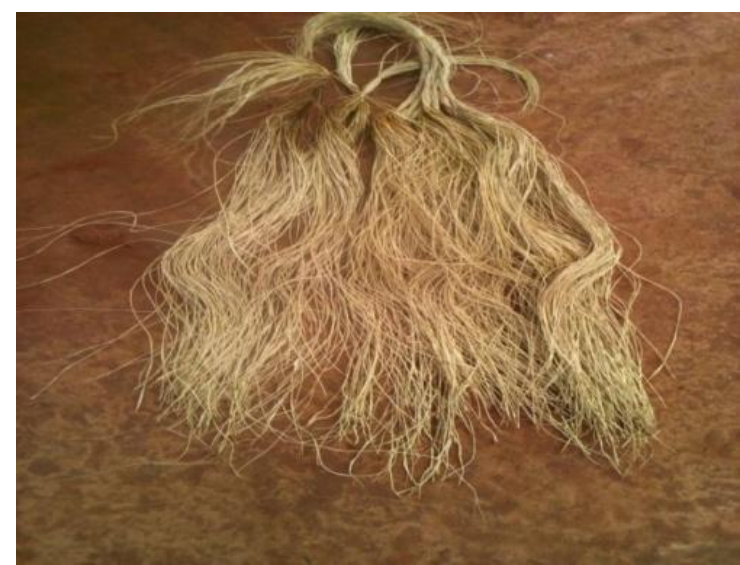

Photo. Fibres extraites des rameaux de Raphia sudanica

Source. Cliché N’Tcha, 2019

En dehors des nattes,il y a les balais appelés «xakun »qui sontobtenus à partir de l'extraction des brindilles des rameaux Raphia sudanica. Unancien pêcheur situe l'usage des rameaux et de leurs fibres à une période lointaine à travers ces propos:

\begin{abstract}
« Nous utilisonsles rameaux et leurs fibres pour faire des filets à cause de leur résistance. Les filets qu'on utilise pour pêcher certains types de poissons. On tisse ces filets et on les positionne au bord de la rivière avant de mettre les anneaux. Comme il n'y avait pas de ficelles en textiles, c'est avec les rameaux de raphia et leurs fibres que les gens capturaient les poissons et comme les blancs ont amené les ficelles maintenant, c'est pourquoi on ne les voit plus trop utiliser les rameaux et leurs fibres » (Propos de : TZ, Tori-Agonsa le 09/06/2019).
\end{abstract}

Ces propos d'un ancien pêcheur devenu un haut dignitaire religieux permet de comprendre les multiples recours des rameaux de Raphia sudanica par les groupes sociaux locaux. En effet, les rameaux de l'espèce et leurs fibres une fois séchés sont très résistants comme cordes. C'est pourquoi, les communautés recourent à ces organes pour la confection des objets qui en requiert l'usage. C'est ce qui explique qu'on dénombre plus d'une cinquantaine d'objets fabriqués à partir des organes de cette espèce végétale dont au moins une quinzaine comportant les rameaux et/ou leurs fibres. Mais, outre ces usages des rameaux de raphia et de leurs fibres pour la conception de certains objets commercialisés, ces organes de l'espèce sont aussi utilisés dans les pratiques magico-religieux.

\title{
2.2. Usages des rameaux de Raphia sudanica à des fins magico-religieuses
}

Plusieurs concepts font appel aux rameaux dans la tradition chrétienne : Carême, Croix, Dimanche des rameaux, Liturgie, Passion, Rameaux, Semaine Sainte.Mais, "Rameaux"est un mot quelque peu savant pour évoquer une branche d'arbre mais qui d'entrée de jeu provoque des associations (dimanche des rameaux, bénédiction des rameaux, procession des rameaux, rameaux bénits...).

« On emploie plus couramment le mot «palmes » pour désigner les rameaux, mais sans trop le réaliser, on fait alors référence aux rameaux de palmier dans ce cas. $\mathrm{Ne}$ dit-on pas en latin «Dominica in Palmis», alors que les anglophones ont leur «Palm Sunday». L'évangéliste Matthieu fait allusion à des «branches d'arbres », Marc à de la «verdure » et que Luc ignore le détail, Jean évoque explicitement des «rameaux de palmiers »tenus en main pour accompagner les cris de joie l'entrée triomphale de Jésus à Jérusalem. Vue la mention explicite de «palmes » dans l'évangile de Jean, leur usage s'est largement imposé. Certes, les retrouver dans les pays plus nordiques n'est pas sans exotisme mais n'est-ce pas propre à évoquer le souvenir du pays de Jésus. Un rituel chrétien suggère que la croix de procession soit décorée car il ne faut pas la négliger ni surtout l'oublier particulièrement en ce dimanche. Le rituel rappelle d'ailleurs qu'on trouve «en tête le ministre portant la croix ornée d'un rameau». Idéalement, comme le suggère la «Présentation Générale du Missel Romain », cette croix de procession est celle qui, en principe, se retrouve en permanence à proximité de l'autel (Propos de : PD, Adjarra le 09/06/2019). 
Au Bénin, dans les communes du Sud-Est où les palmiers raphia sont plus abondants et facilement accessibles, les fidèles chrétiens ont plus recours aux rameaux de Raphia sudanica pour répondre aux besoins rituels périodiquement. Il convient de faire remarquer que suivant le lieu, l'espèce végétale d'où sont extraits les rameaux diffère; et pour cause les perceptions des plantes par les groupes sociaux locaux qui ont recours à elles et la disponibilité des palmiers.

Enfin, il faut mettre un accent sur la distribution des rameaux qui sont souvent offerts par les paroisses. Des bénévoles fractionnent les palmes et les présentent aux participants lors de l'accueil. Un geste qui requiert un soin particulier parce qu'il crée un lien entre la communauté et la maison du fait qu'une fois la célébration terminée, on aime en rapporter chez soi et les conserver l'année durant. Dans un beau geste catéchétique, on peut à l'occasion s'y référer.

Par ailleurs, les rameaux de Raphiasudanica sont utilisés par les groupes sociaux locaux du Sud-Est du Bénin à des fins magico-religieuses. A ce propos, un haut dignitaire religieux rapporte que :

«Les rameaux de raphia sont utilisés pour protéger les bouteilles dans leșiquelles se trouvent certaines recettes magiques. Certaines bouteilles sont protégéeś par les rameux séchés et travaillés pour que personne ne puisse voir ni détrúíire leur contenu à travers les bouteilles. Celle qui est là, on l'appelle «adjaǵfodoe» » (Propos de : TZ, Tori-Agonsa le 09/06/2019).

Il ressort de ces propos que certaines personnes utilisent les rameaux de Raphia sudanica sur la base des croyances qu'ils ont quant aux vertus de cet organe de l'espèce. La photo ci-dessous est celle d'une bouteille à potion magique protégée par les rameaux séchés et travaillés à cet effet.

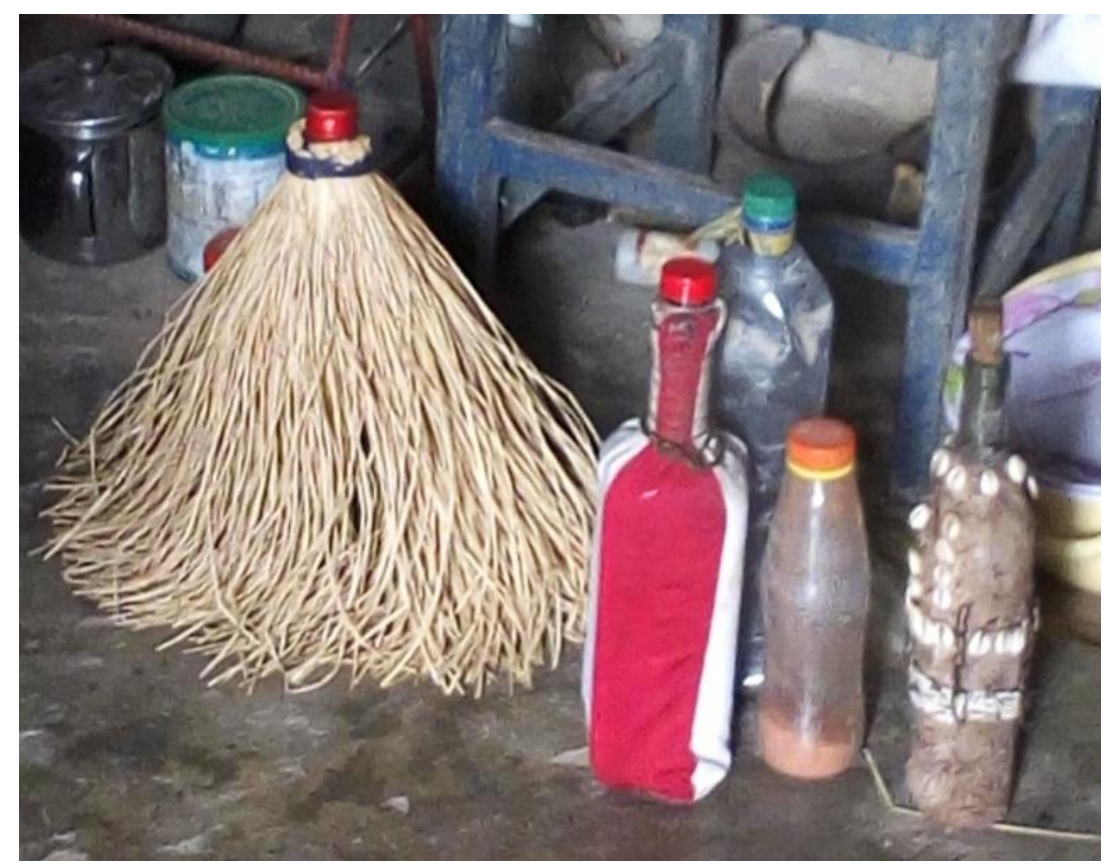

Photo. Bouteille à potion magique couverte de rameaux de Raphia sudanica

Source. Cliché N’Tcha, 2019

Ce sont d'ailleurs ces croyances aux vertus magiques des rameaux de cette plante qui font que plusieurs objets confectionnés à partir de ces organes sont utilisé à des fins magico-religieuses. Un tradi-praticien ajoute à propos de ces croyances religieuses que :

«Il y a un sac appelé« adókpoó » ou «chakoto» fabriqué avec les rameaux de raphia, que les vieux utilisent. C'est dans ce sac que ces vieux rangent leurs recettes magiques. Les rameaux de raphia possèdent une puissance infranchissable qui empêche les ennemis d'atteindre spirituellement la personne qui met ses recettes magiques de protection dans ce sac. C'est pourquoi il est défendu à quiconque de toucher à ce type de sac sans l'autorisation de son propriétaire, surtout quand il s'agit d'une personne de sexe féminin (notamment en menstruations). Cela permet de préserver leurs vertus protectrices» (Propos de : FT, ToriAgonsale09/06/2019). 
Ces différents discours permettent de se rendent compte que les croyances des groupes sociaux locaux aux forces spirituelles des rameaux,et par-delà, que tous les organes de Raphia sudanica, occupent une place de choix dans leur vision du monde. C'est en témoignage du respect et à la reconnaissance de l'étendue de la puissance de la divinité «Zãngbetó » que les adeptes de cette divinité recourent aux rameaux de l'espèce pour fabriquer la case servant lors de la sortie de la divinité. Par conséquent, les adeptes de la divinité «Zãngbetó » utilisent les feuilles, rameaux et fibres de cette espèce pour fabriquer la case qui lui permet d'apparaître en publique. L'affirmation de cet interlocuteur va plus dans le détail.

«On exploite les rameaux, on extrait les fibres « od $\varepsilon$ », ce que les femmes utilisent pour tresser les nattes; on enlève maintenant les brindilles et le reste appelé «dexuixa» permet de fabriquer la case de Zãngbetó» (Propos de : FT, ToriAgonsa le 09/06/2019).

Cette affirmation est soutenue par celle d'un adepte de «Zãngbetó» qui apprend que :

«C'est les feuilles ou les rameaux «dexuixa » et leurs fibres qu'on utilise pour construire la case de «Zãngbstó ». C'est le « djôwanou» de nos aïeux ; c'est ça les gens avaient utilisé pour faire peur à un roi et il a arrêté la guerre. C'est à cause de ça que le roi Toffa est venu à Porto-Novo. Ce sont nos grands-parents qui nous ont raconté l'histoire. C'est la fibre et le « dexuixa »qu'on utilise pour fabriquer les cases de cette divinité » (Propos de : TA, ToriAgonsa le 09/06/2019).

Le choix des organes de Raphia sudanica pour la fabrication des cases de la divinité n'a pas été fait de façon hasardeuse. En réalité, les groupes sociaux locaux considère cette plante comme étant la plus résistante de toutes, et donc relevant de l'ordre divin. C'est pourquoi ces organes sont choisis pour montrer la place accordée à la divinité par ces adeptes. La photo suivante est celle d'une case de la divinité « Zãngbetó».

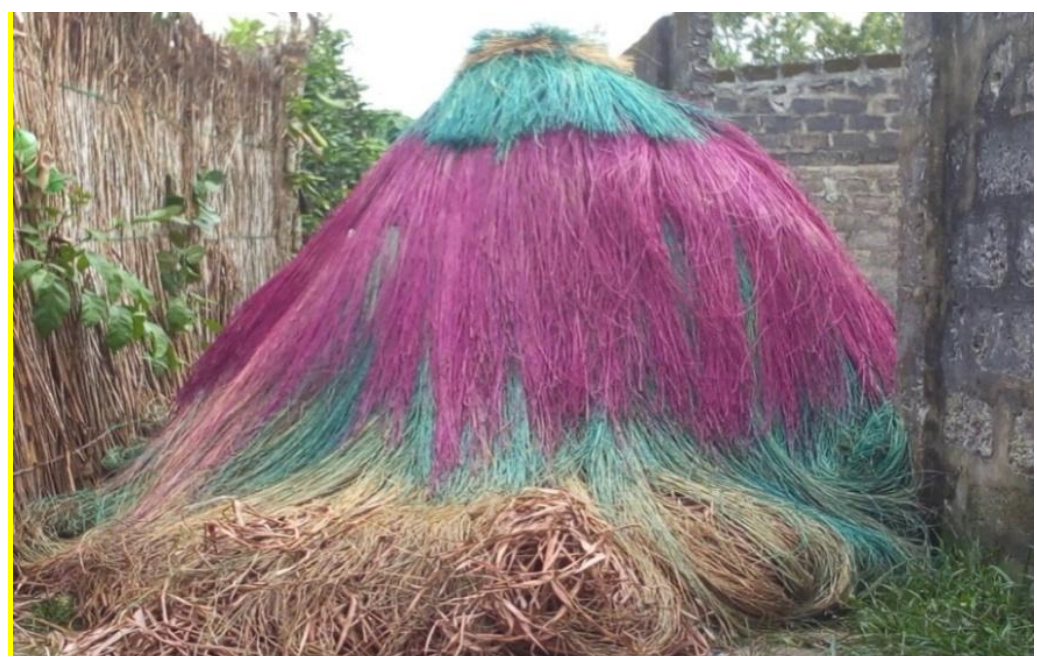

Photo. Cage de la divinité Zãngbetó

Source. Cliché N'Tcha, 2019

C'est dans cette case que la divinité apparaît en publique pour manifester son pouvoir et sa puissance lors des cérémonies, en produisant des choses magiques pour montrer l'étendue de la force spirituelle.

Les usages sociaux ressortis dans ce travail ne sont pas exhaustifs. Il y a des pratiques faisant appel aux rameaux de Raphia sudanica qui ne sont pas apparus ici, mais qui démontre de la multiplicité des usages sociaux de cette espèce par les groupes sociolinguistiques du Sud-Est du Bénin. En ce qui concerne les croyances en la force spirituelle des organes de cette plante, elles ne sont pas exclusivement observables chez les adeptes des divinités qui ont des rapports directs avec l'espèce mais ces croyances s'étendent jusqu'aux membres des groupes sociaux locaux.

En effet, les rameaux véhiculent des messages selon leur emplacement dans l'environnement humain. Les membres des groupes sociaux locaux ont connaissent du code langagier véhiculé par la présence des rameaux de Raphia sudanicaà un endroit précis. C'est pourquoi, certaines personnes usent de ces rameaux pour sécuriser leurs biens matériels dans ces localités. La présence d'un rameau séché de 
raphia attaché à l'entrée d'un champ, signale l'interdiction d'accès à ce dernier et le contrevenant risque de subir les conséquences émises par son propriétaire auprès de la divinité représentée par ce rameau.

Ainsi, il est courant de voir dans ces localités, les rameaux de Raphia sudanica sur les ailes des volailles, les cous desbétails, dans les bassines de céréales et d'autres biens matériels marquant de ce fait la sacralité de ceux-ci. Un chef culte explique que :

«Si par exemple quelqu'un vole nos biens chaque fois et se montre tout puissant, si on met un rameau ou sa fibre sur quelque chose et la personne prend, même s'il va au Vatican, il va revenir nous rendre compte et c'est forcé » (Propos de : KA, Dangbo le 24/06/2019).

Les rameaux de Raphia sudanica participent à la sacralisation des biens dans les localités du Sud-Est du Bénin et les membres des groupes sociaux locaux respectent ces normes et valeurs endogènes qui participent au maintien de l'ordre social dans le milieu.

\subsection{Usages des rameaux de Raphia sudanica à des fins thérapeutiques}

Les rameaux de Raphia sudanicasont utilisés lors des purifications des individus et dans les recettes destinées à la guérison de certaines maladies. Les rameaux sont connus pour être utilisés par les fidèles du christianisme céleste lors des cérémonies de purification des fidèles pour les débarrasser de leur impureté. Un fidèle de cette confession religieuse explique le choix porté sur les rameaux comme éléments constitutif utilisé lors de ce rituel.

«Le choix des rameaux de raphia remonte à la célébration du christ lors de son entrée dans la ville de Jérusalem et la fête des rameaux fait référence à ce moment. Ailleurs, les gens n'utilisent pas forcément les rameaux de raphia ni de palmier, mais ceux des plantes qu'ils ont sous la main et qui représente une plante pure à leurs yeux en fonction des réalités de leur milieu. Chez nous le raphia est considéré comme une plante pure et c'est à cause de leur virginité que l'on trouve aux rameaux de cette plante, que l'on a recours à elle lors de cette fête des rameaux pour purifier les fidèles de l'église» (Propos de : RK, Djrègbé le 08/06/2019).

Cette explication fournie par un fidèle de l'église du christianisme céleste renseigne sur les fondements qui sont à la base de l'utilisation des rameaux lors de la purification des fidèles de cette église. Mais, cette forme de purification n'est pas utilisée qu'à l'église, les thérapeutes traditionnels utilisent aussi les rameaux de cette espèce lors des purifications qu'ils opèrent sur les membres des défunt(e)s dont la survenance de la mort nécessite une purification des autres membres de leurs familles encore en vie. La purification des objets avec lesquels ces défunt(e)s ont été en contact, est souvent faite pour éviter que le malheur ne continu de décimer la famille pour une faute commise par le ou la défunt(e). Les rameaux sont utilisés pour asperger l'eau destinée à cet effet sur les personnes concernées, les objets et dans les chambres de la personne décédée.

Par ailleurs, les rameaux de raphiasont utilisés pour soigner l'ulcère gastrique ou ceux qui ont une température élevée dans leur ventre. Ils sont sollicités aussi dans la recette qui permet de guérir les maux d'yeux notamment «les petites boules qui restent sur les yeux et empêchent parfois de voir de loin» selon les propos de: KA, Dangbo le 24/06/2019. Le filtrat des rameaux de Raphia sudanicapermet de lutter contre la tensionartériellechez les personnes qui en souffrent. Il est aussi rapporté que :

«Lorsque quelqu'un dort sur son cou et ressent des douleurs au niveau du cou, on donne les fibres de raphia à une personne gauchère qui prend le soin de les tresser. Ensuite, on attache cette tresse des fibres au cou du malade. Le mal va partir » (Entretien réalisé en ouémè, IDKDénankpo le 12/06/2019).

Les usages sociaux des rameaux de cette plante à des fins thérapeutiques sont multiples et diverses. Les groupes sociaux locaux nourrissent des croyances autour de l'espèce qui orientent leurs comportements vis-à-vis de ses organes. C'est donc sur cette base que sont collectés et utilisés les rameaux de Raphia sudanicadans les différentes localités du Sud-Est Bénin. 


\section{DISCUSSION}

Les usages sociaux des rameaux de Raphia sudanicaont un impact sur sa conservation dans les localités du Sud-est Bénin. En effet, tels que utilisés par les groupes sociaux locaux, aucun contrôle n'est effectué autour de la collecte des organes de l'espèce pour une exploitation durable. En effet, les activités anthropiques constituent l'un des défis les plus importants auxquels est confrontée la conservation des ressources naturelles. Les populations s'accroissent plus vite et deviennent nombreuses que les ressources peuvent en supporter. Les initiatives communautaires sont envisagées pour alléger les effets de la dégradation de l'environnement comme le fait remarquer M. Mahdi (2012), mais ces efforts sont loin des dommages créés. En ce qui concerne les ressources forestières, aucune espèce végétale n'est en marge de la sollicitation de l'Homme. Si l'on regarde dans le passé, l'on voit que quelques familles de plantes ont joué un rôle prédominant en tant que source de matières premières à la fois comestibles et non comestibles. Dans le monde entier, trois familles de plantes se démarquent en terme d'utilité, tant par le passé qu'aujourd'hui: la famille des graminées (Gramineae), la famille des légumineuses (Leguminosae) et la famille des palmiers (Palmae). Si la zone géographique considérée est restreinte aux régions tropicales, l'importance de la famille des palmiers est encore plus flagrante (Johnson, 2010).Cela situe les usages des palmiers à une période lointaine et permet de rejoindre cette affirmation deM. OuassaKouaro (2011) selon laquelle «l'utilisation des différentes parties de l'arbre démontre le rôle essentiel qu'il tient dans la vie des groupes sociaux » (M. OuassaKouaro, 2011, p. 61).

Les rameaux de Raphia sudanica font parties des organes de l'espèce les plus utilisés par les groupes sociaux locaux. D'ailleurs, les rameaux de palmiers ont souvent fait partie des composantes les plus importantes intervenant dans les rituels sacrés des religions. Ainsi, si dans le Nord-ouest du Bénin, les ressources ligneuses représentent un levier du développement économique comme le soutiennent $\mathrm{M}$. OuassaKouaro et $\mathrm{O}$. N. Tchoroué (2015), dans d'autres contrées ce sont les ressources forestières non ligneuses qui jouent ce rôle. Au Sud-Est du Bénin, Raphia sudanica est une ressource naturelle qui tient une place de choix dans la vie de plusieurs groupes sociaux locaux et le maintien de l'accès à cette ressource est d'autant plus important qu'il y ait lieu de se préoccuper de sa conservation. Car, confrontés aux impératifs du quotidien, les membres de ces groupes sociaux n'ont parfois d'autres choix que d'innover avec les ressources disponibles pour subvenir à leurs besoins. Les usages sociaux des espèces végétales sont en perpétuels augmentation.

En dehors des usages sociaux de rameaux de Raphia sudanica par les groupes sociaux locauxqui ont été décrits dans ce travail, il importe de faire remarquer que cette plante participent aussi à l'entretien de plusieurs activités surtout génératrices de revenus dans le Sud-Est du Bénin. De fait, elle constitue pour 38\% des acteurs approchés une matière première parce que toute leur activité est basée sur ses organes. Ce fait est loin d'être un fait nouveau car son importance a été déjà démontrée par les travaux de F. Meutchieye (2012) à l'Ouest du Cameroun. Il est donc important d'accompagner les usages sociaux des ressources naturelles par des mesures de conservation adéquates pour s'assurer de leur pérennité et celle des activités qui en dépendent.

\section{CONCLUSiON}

Cette recherche s'est intéressée à la description des usages sociaux des rameaux de Raphia sudanicadans le Sud-Est du Bénin. Elle a adopté une démarche qualitative en s'appuyant sur l'analyse des données documentaires, des entretiens approfondis réalisés avec les groupes cibles et des observations directes faites sur le terrain. L'on retient de cette recherche que l'usage des rameaux de Raphia sudanica par les groupes sociaux locaux du Sud-Est du Bénin ne s'éloigne pas des formes d'usages sociaux des rameaux observées dans les autres contrées du monde. Les usages sociaux des organes de cette espèce sont multiples : les hommes comme les femmes ont recours aux organes de cette espèce pour leurs activités artisanales (pêche, vannerie, tressage, fabrication de vin...), les tradithérapeutes pour guérir des maladies et éviter la survenance du mal ou de la mort chez certains patients, les religieux pour les rituels de purification. 
Au Bénin, nombreux sont les milieux dans lesquels les palmiers sont les plantes dont les rameaux servent de constituants pour l'exécution de ce rituel. Dans le Sud-Est du Bénin, où la recherche a été menée, les palmiers sauvages, notamment les raphias sont majoritairement les plantes qui peuplent les forêts de ce milieu. Par conséquent, les groupes sociaux locaux recourent à elles pour des usages destinés à des fins multiples et diversifiés. Les rameaux de Raphia sudanica sont utilisés dans les activités destinées à des fins économiques. Les artisans ont recours à eux pour des transformations afin de compléter les œuvres à commercialiser. Cet aspect de l'usage des rameaux de cette espèce végétale n'est pas le seul observable dans ce milieu, car ils sont aussi utilisés à des fin magicoreligieux par les mêmes groupes sociaux riverains. Ainsi, retrouve-t-on les rameaux ou leurs extraits dans les pratiques religieuses, témoignant de ce fait des croyances religieuses qui se nourrissent autour de cette espèce, de ses organes et plus précisément des rameaux qui en sont issus. Les groupes sociaux locaux exploitent Raphia sudanicaà des fins thérapeutiques également. Car, les vertus de cette plante s'étendent au domaine médicinal. Qu'elles soient considérées comme pratiques magico-religieuses ou pratiques traditionnelles de la médecine, ce qui est remarquable dans le contexte actuel, c'est que les groupes sociaux qui ont à faire à cette plante croient et utilisent ses rameaux pour guérir des maladies ou se débarrasser du mal.

Ces divers usages sociaux des rameaux dans le Sud-Est du Bénin ne sont pas sans conséquences sur son exploitation. L'usage des organes d'une plante sous-tend leur collecte et donc son exploitation, qui n'est pas toujours contrôlée et gérée rationnellement par les groupes sociaux riverains pour en assurer sa durabilité dans le milieu. Les populations s'accroissent continuellement et par-là les demandes de produits confectionnés à base de rameaux de Raphia sudanica. De plus, les pratiques sociales, culturelles, religieuses et thérapeutiques pour lesquelles les rameaux de l'espèce sont sollicités ne font qu'augmenter l'ampleur des prélèvements des organes de l'espèce, montrant ainsi la pression à laquelle elle est soumise dans le milieu. Les exploitations de cette forme ne sont pas toujours mises en exergue pour stimuler des réflexions projetant les actions anthropiques présentes à des effets probables sur le couvert végétal dans le long terme. Car si Raphia sudanicaest compté encore parmi les espèces végétales du Sud-Est du Bénin, il est certain que sa population n'est plus la même aujourd'hui qu'elle ne l'a été par le passé et augure de sa disparition certaine si des politiques publiques ne mènent pas des actions pour son exploitation durable au vu des possibilités qu'il offre dans la sécurité alimentaire et la survie des communautés dans une démarche inclusive.

\section{REFERENCES}

[1] Assogbadjo, A. E., Glèlè Kakaï, R., Vodouhê, F., Djagoun, C. A., Codjia, J. T., \& Sinsin, B. (2012). Biodiversity and socioeconomic factors supporting farmers' choice of wild edible trees in the agroforestry systems of Benin (West Africa). Forest Policy and Economics, pp. 41-49.

[2] Descola, P. (2015). Par-delà nature et culture. (c. F. essais, Éd.) Paris: Gallimard.

[3] Dongmo, D. M. (n.d.). Etudes floristiques et ethnobotaniques dans un village de la zone forestière du Caeroun: cas de NKobibanda. Yaoudé, Cameroun: Université de Yaoudé I.

[4] Ernst, C. E. (2012). Cartographie du couvert forestier et des changements du couvert forestier en Afrique central.

[5] INSAE [Institut National de la Statistique et de l'Analyse Economique]. (2013). Cahier des villages et quartiers de villes. Cotonou: République du Bénin.

[6] Johnson. (2010). Les palmiers tropicaux. Produits Forestiers Non Ligneux 10/Rev. 1. Rome: FAO.

[7] Lacaze, L. (2013). Interactionnisme de Blumer revisité. De Boeck Supérieur "Sociétés", 41-52.

[8] Mahdi, M. (2012, Décembre 31). Initiatives communautaires pour la gestion durable des forêts dans le haut atlas marocain. In : Ba Mbow A. F. et al. (2012). Désertification et réponses paysannes, Edition : AGRIDAPE, 28(3), pp. 22-23.

[9] Meutchieye, L. (2012, Décembre 31). Quelles stratégies de conservation des eaux de surface dans les régions montagneuses de l'Ouest Caméroun? Initiatives autour des raphiales. In : Ba Mbow, A. F. et al. (2012). Désertification et réponses paysannes, pp. 26-27.

[10] Michel Arnold, J. E., \& Ruiz Perez, M. (2001, Août 6). Can non-timber forest products match tropical forest conservation and development objectives? Ecological economics, pp. 437-447.

[11] Ouassa Kouaro, M. (2011). Usages sociaux du néré (Parkia biglobosa) dans le Nord du Bénin. Annales de la Faculté des Lettres, Arts et Sciences Humaines, 2(17), pp. 56-71.

[12] Ouassa Kouaro, M., \& Tchoroué, N. O. (2015). Enjeux socioculturels de la gestion des bois sacrés dans le nord-ouest Bénin. Cahier du CBRST, 3(8), pp. 
[13] PNUD [Programme des Nations Unies pour le Développement]. (2013). Objectifs du Millénaires pour le Développement. New York: Nations Unies, 64p

Citation: Dr Ludovic K. N'TCHA, Dr Monique OUASSA KOUARO. "Usages Sociaux Des Rameaux Et Exploitation Durable De Raphia Sudanica Dans Le Sud-Est Du Benin” International Journal of Humanities Social Sciences and Education (IJHSSE), vol 7, no. 12, 2020, pp. 64-74. doi: https://doi.org/10.20431/23490381.0712008 .

Copyright: (C) 2020 Authors. This is an open-access article distributed under the terms of the Creative Commons Attribution License, which permits unrestricted use, distribution, and reproduction in any medium, provided the original author and source are credited. 